\title{
Pseudohalogenometallverbindungen, LXV [1] Synthese von Tetrazolen und Triazolen über die 1,3-dipolare Cycloaddition an die Azid-Liganden von polymeren Cobalt(III)- und Palladium(II)-Komplexen. Darstellung und Struktur von 5-Trichlormethyltetrazol
}

\author{
Pseudohalogeno Metal Compounds, LXV [1] \\ Synthesis of Tetrazoles and Triazoles via 1,3-Dipolar \\ Cycloaddition to the Azido Ligands of Polymeric Cobalt(III) and Palladium(II) Complexes. \\ Synthesis and Structure of 5-Trichloromethyltetrazole \\ Josef Geisenberger, Jürgen Erbe, Jürgen Heidrich, Ulrich Nagel ${ }^{\text {a }}$ und Wolfgang Beck* \\ Institut für Anorganische Chemie der Universität München, Meiserstraße 1, D-8000 München 2 \\ Herrn Professor Dr. Drs. h. c. Oskar Glemser zum 75. Geburtstag gewidmet \\ Z. Naturforsch. 42b, 55-64 (1987); eingegangen am 24. Juli 1986 \\ Tetrazoles, Triazoles, Polymeric Schiff Base Cobalt(III) Complexes, \\ Phosphine Palladium Complexes with Azide, Tetrazolate and Triazolate Ligands \\ The cycloaddition of nitriles and of dimethylacetylenedicarboxylate to the azide ligand of \\ polymeric Schiff Base cobalt(III) and phosphine palladium(II) complexes gives the corresponding \\ tetrazolate and triazolate complexes from which the heterocycles could be cleaved by hydrogen \\ chloride. Usually the yields are low; if the heterocycle is soluble in ether or sublimable, yields up \\ to $30 \%$ have been obtained. Using this method the hitherto unknown 5-trichlormethyltetrazole \\ could be prepared which was characterized by an X-ray structural analysis. Similarly, the cyclo- \\ addition of azido(tetraphenylporphinato)cobalt(III) with nitriles, cyclohexylisocyanide and \\ $\mathrm{MeO}_{2} \mathrm{CC} \equiv \mathrm{CCO}_{2} \mathrm{Me}$ affords the corresponding complexes with heterocyclic ligands. The prepa- \\ ration of tetraphenylporphyrinato(tricyanmethanido)cobalt(III), (TPP) CoN $=\mathrm{CC}(\mathrm{CN})_{2}, \quad$ is \\ reported.
}

\section{Einleitung}

An den koordinierten Azid-Liganden verschiedener Übergangsmetall-Komplexe lassen sich Mehrfachbindungssysteme unter Bildung von Heterocyclen-Komplexen addieren [2-4]. Diese 1.3-dipolaren Cycloadditionen nach Huisgen [5] verlaufen mit Azido-Metall-Komplexen häufig unter überraschend milden Bedingungen. Aus Tetrazolato- und Triazolato-Komplexen ließen sich mit Säuren oder Säurechloriden in einigen Fällen die neutralen Heterocyclen freisetzen [2-4, 6, 7]. Zur Darstellung von Heterocyclen nach dieser Methode bieten sich polymere, schwerlösliche Azido-Komplexe an, die eine Trennung Komplex/Heterocyclen erleichtern sollten. Über einige polymere Schiffbase-Cobalt-Komplexe sowie polymere Phosphan-haltige Azido-PalladiumKomplexe haben wir kurz berichtet [8].

\footnotetext{
* Sonderdruckanforderungen an Prof. W. Beck.

a Röntgenstrukturanalyse.

Verlag der Zeitschrift für Naturforschung, D-7400 Tübingen $0340-5087 / 87 / 0100-0055 / \$ 01.00 / 0$
}

\section{Ergebnisse und Diskussion}

Reaktionen mit polymeren Schiffbase-Cobalt(III)Komplexen

Cobalt-Komplexe mit polymeren Schiffbase-Liganden aus 5,5'-Methylen-bis(salicylaldehyd) und Diaminen sind schon länger bekannt [9]. Als Ausgangsverbindungen setzten wir die Azido-Komplexe $1 \mathbf{a}, \mathbf{b}$ ein, die nach Sawodny und Riederer [10] erhalten wurden. Monomere Komplexe $\mathrm{Co}^{\text {III }}$ (chelat) $\mathrm{N}_{3}$ $[11,12]$ addieren bereitwillig Acetylene und Nitrile [4]. Ebenso setzen sich $\mathbf{1 a}, \mathbf{b}$ mit verschiedenen Nitrilen zu den polymeren Tetrazolato-Komplexen 2 um. Wie mit organischen Aziden [5] vollzieht sich die Cycloaddition der Nitrile an die koordinierte Azidgruppe um so rascher, je elektronenärmer das Nitril ist. So reagiert Trichloracetonitril bereits bei Raumtemperatur innerhalb von $14 \mathrm{~d}$ quantitativ zu 2 a. Es ergab sich, wie durch die Abnahme der Intensität der IR $-\mathrm{N}_{3}$-Bande festgestellt wurde, folgende Abstufung der Reaktivität der Nitrile $\mathrm{CCl}_{3} \mathrm{CN}>$ $\mathrm{NCCH}_{2} \mathrm{CN}>\mathrm{NC}\left(\mathrm{CH}_{2}\right)_{2} \mathrm{CN}>p-\mathrm{C}_{6} \mathrm{H}_{4}(\mathrm{NO})_{2}(\mathrm{CN})>$ $o-\mathrm{C}_{6} \mathrm{H}_{4}(\mathrm{CN})_{2} \approx m-\mathrm{C}_{6} \mathrm{H}_{4}(\mathrm{CN})_{2} \approx p-\mathrm{C}_{6} \mathrm{H}_{4}(\mathrm{CN})_{2}>$ $\mathrm{CH}_{3} \mathrm{CN}>\left(\mathrm{CH}_{3} \mathrm{O}\left(\mathrm{CH}_{2}\right)_{2} \mathrm{CN}\right)$. Der Azido-Komplex 1 a reagiert wesentlich rascher als der durch die Ami- 
nogruppe koordinativ gesättigte Komplex 1b. Bei monomeren $\mathrm{Co}$ (chelat)(5-R-tetrazolat)-Komplexen wurde festgestellt, daß die $\mathrm{N}(2)$-Koordination des Tetrazolats aus sterischen Gründen begünstigt ist $[6,7]$. Ebenso liefert die Cycloaddition des AzidIons an das koordinierte Nitril in $\left[\left(\mathrm{H}_{3} \mathrm{~N}\right)_{5} \mathrm{CoNCR}\right]^{3+}$ das N(2)-Isomere als thermodynamisch stabiles Produkt [13]. Daher nehmen wir an, daß auch in 2-7 der Tetrazolat-Ligand über N(2) an das Metall koordiniert ist.

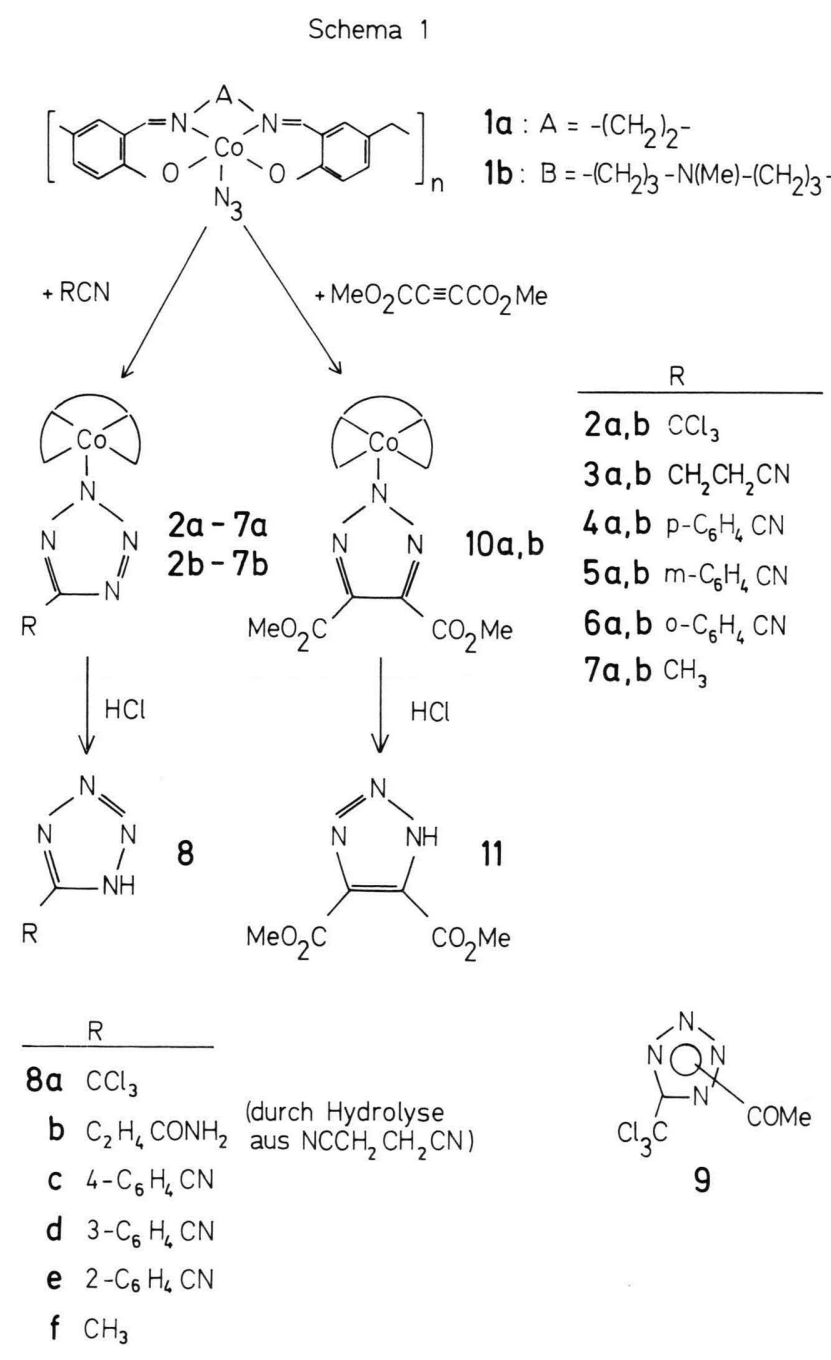

Die freien 5-R-Tetrazole lassen sich aus 2-7 mit Chlorwasserstoff in Diethylether oder Methanol abspalten. Durch teilweise Zersetzung der polymeren Komplexe 2-7 ist die Ausbeute in den meisten Fällen sehr gering. Wenn jedoch das Tetrazol leicht löslich oder sublimierbar ist, kann die Ausbeute ge- steigert werden. So konnte auf diese Weise erstmals 5-Trichlormethyltetrazol 8a (Ausbeute ca. 30\%) gefahrlos dargestellt werden [14]. Die beim Versuch der Darstellung von $8 \mathbf{a}$ aus $\mathrm{CCl}_{3} \mathrm{CN}$ und Alkaliazid beobachtete Explosion [15] war möglicherweise durch Substitution der Chlor-Substituenten der $\mathrm{CCl}_{3}$-Gruppe durch Azid verursacht, wobei sehr instabile Verbindungen entstehen sollten. Eine solche Reaktion ist nach dem hier beschriebenen Verfahren nicht möglich, da kein überschüssiges Azid vorhanden ist; nur das koordinierte Azid kann mit Nitrilen zum Tetrazol abreagieren. 8a explodiert beim langsamen Erhitzen bis zum Schmelzpunkt $\left(\mathrm{N}_{2}\right.$-Entwicklung) nicht. Die bei der Umsetzung von $\mathbf{2 a}, \mathbf{b}$ mit $\mathrm{HCl}$ entstehenden Chloro-Cobalt-Komplexe lassen sich mit $\mathrm{NaN}_{3}$ wieder zu $\mathbf{1 a}$, b regenerieren, die dann für eine erneute Cycloaddition zur Verfügung stehen. Im Prinzip entstehen dann die Tetrazole aus RCN, $\mathrm{HCl}$ und $\mathrm{NaN}_{3}$ :

$$
\begin{array}{ll}
\mathrm{Co}(\text { chelat }) \mathrm{N}_{3}+\mathrm{RCN} & \rightarrow \mathrm{Co}(\text { chelat }) \mathrm{N}_{4} \mathrm{CR} \\
\mathrm{Co}(\text { chelat }) \mathrm{N}_{4} \mathrm{CR}+\mathrm{HCl} & \rightarrow \mathrm{Co}(\text { chelat }) \mathrm{Cl}+\mathrm{HN}_{4} \mathrm{R} \\
\mathrm{Co}(\text { chelat }) \mathrm{Cl}+\mathrm{NaN}_{3} & \rightarrow \mathrm{Co}(\text { chelat }) \mathrm{N}_{3}+\mathrm{NaCl} \\
\hline \mathrm{RCN}+\mathrm{NaN}_{3}+\mathrm{HCl} & \rightarrow \mathrm{HN}_{4} \mathrm{CR}+\mathrm{NaCl}
\end{array}
$$

Bei der Umsetzung von 2a mit Benzoyl- und Acetylchlorid konnten die $\mathrm{N}$-acylierten 5-Trichlormethyltetrazole IR- und ${ }^{1} \mathrm{H}-\mathrm{NMR}$-spektroskopisch nachgewiesen werden. Der Isolierung der Heterocyclen steht die Zersetzung der Chelat-CobaltKomplexe durch Alkyl- und Acylhalogenide entgegen. N-Acetyl-5-trichlormethyltetrazol 9, das wie andere N-Acyl-5-R-Tetrazole [16] auch direkt aus 8a und Acetylchlorid erhalten wurde, entsteht dabei als Gemisch der N(1)- und N(2)-Isomeren. Dagegen führt die Alkylierung von monomeren Co(chelat)(tetrazolat)-Komplexen regiospezifisch zu N(1)-substituierten Tetrazolen $[6,7]$.

Analog wurden 1a, b mit Acetylendicarbonsäuredimethylester zu den Triazolato-Komplexen 10a, b umgesetzt, aus denen mit $\mathrm{HCl} /$ Ether das freie Triazol 11 gewonnen wurde. Der Triazolat-Ligand in $\mathbf{1 0}$ ist vermutlich wie in monomeren Komplexen [4, 12] über N(2) an das Metallatom gebunden.

\section{Reaktionen mit Azido(tetraphenylporphinato)- cobalt(III)}

Im Zuge dieser Arbeiten wurde auch Azido(tetraphenylporphinato)cobalt(III) (13) mit $\mathrm{CH}_{3} \mathrm{CN}$ und $\mathrm{CCl}_{3} \mathrm{CN}$ sowie Acetylendicarbonsäuredimethyl- 
Schema 2

$$
\mathrm{Co}(\mathrm{TPP}) \mathrm{CH}_{3}{ }^{19)} \frac{\mathrm{HBF}_{4} \cdot \mathrm{Et}_{2} \mathrm{O}}{-\mathrm{CH}_{4}}
$$

TPP = Tetraphenylporphinato<smiles>CC(=O)OC1=NNNN1[PH3-]</smiles>

14

$$
[\mathrm{Co}(\mathrm{TPP})]^{+} \mathrm{BF}_{4}^{-} \quad \underset{-\mathrm{BF}_{4}^{-}}{\stackrel{+\mathrm{N}_{3}^{-}}{\longrightarrow}} \mathrm{Co}(\mathrm{TPP}) \mathrm{N}_{3}
$$

12

13

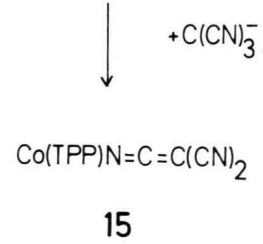

$$
\begin{aligned}
& \left.m \mathrm{PPh}_{2}\right)_{2} \mathrm{Pd}\left(\mathrm{N}_{3}\right)_{2} \\
& 16 \mathrm{a} / \mathrm{b} \\
& m={ }_{\mathrm{C}}^{\mathrm{C}} \mathrm{H} \mathrm{H}_{2}-\mathrm{CH}_{2}-
\end{aligned}
$$

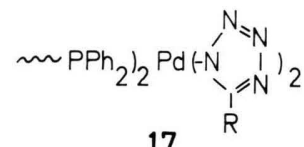

17<smiles>COC(=O)C1=NN([PbH]c2ccccc2)NN1C(C)=O</smiles>

18 ester umgesetzt. Mit Cyclohexylisocyanid entsteht der Tetrazolato-Komplex $\mathbf{1 4}$ mit Co-C-Bindung [17]. Aus den Heterocyclen-Komplexen lassen sich, wie spektroskopisch nachgewiesen wurde, die entsprechenden Tetrazole und Triazole abspalten (vgl. Exper. Teil). $\mathrm{Co}(\mathrm{TPP}) \mathrm{N}_{3}$ (13) erhielten wir nach Schema 2. Der Aqua-Komplex $\mathrm{Co}(\mathrm{TPP})\left(\mathrm{H}_{2} \mathrm{O}\right) \mathrm{N}_{3}$ wurde früher beschrieben [18] und reagiert wie $\mathbf{1 3}$ mit Dipolarophilen.

In 12 ist das Tetrafluoroborat-Ion nicht koordiniert [20]; im IR-Spektrum erscheint die $v \mathrm{BF}_{4^{-}}$ Absorption des freien $\mathrm{BF}_{4}{ }^{-}$-Ions bei $1050 \mathrm{~cm}^{-1}$. 12 reagiert mit stärker koordinierenden Anionen wie Tricyanmethanid $\mathrm{zu} \mathrm{Co}(\mathrm{TPP}) \mathrm{N}=\mathrm{C}=\mathrm{C}(\mathrm{CN})_{2} \quad$ (15), das wir wie $\mathrm{Fe}(\mathrm{TPP}) \mathrm{NCC}(\mathrm{CN})_{2}$ [21] als Dicyanketeniminato-Komplex formulieren.

\section{Reaktionen mit polymeren Diazido-bis(phosphan)- palladium(II)-Komplexen}

Mit dem - aus chlormethylierten Polystyrol (Merrifieldharz) und $\mathrm{KPPh}_{2}$ erhaltenen - polymeren Diphenylbenzylphosphan [22] wurde der AzidoKomplex 16 dargestellt. Dieser reagiert wieder mit Nitrilen und Acetylendicarbonsäuredimethylester zu den Komplexen 17, 18. Bei diesen heterogenen Reaktionen sind zur vollständigen Umsetzung höhere Temperaturen notwendig als mit monomeren $\mathrm{Pd}^{\mathrm{II}}$ Komplexen [2] in homogener Phase.
Auch hier gelingt in einigen Fällen die Abspaltung der Heterocyclen mit $\mathrm{HCl}$ bzw. Acetylchlorid (vgl. Exper. Teil).

\section{Röntgenstrukturanalyse von 5-Trichlormethyl- tetrazol (8a)}

Abb. 1 zeigt eine Ansicht des Moleküls 8a, Tab. I enthält die Bindungslängen und Bindungswinkel. Der Tetrazolring in $\mathbf{8 a}$ ist einschließlich der Substituenten $\mathrm{C}_{2}$ und $\mathrm{H}_{1}$ planar ( $\mp 2 \mathrm{pm}$ ). Alle Bindungslängen im Ring liegen zwischen einer Einfach- und Doppelbindung, was auf eine starke Delokalisierung hindeutet. Dabei ist wie in allen bekannten TetrazolStrukturen die Bindung $\mathrm{N}_{2}-\mathrm{N}_{3}$ am kürzesten [23]. Der Einfluß des Wasserstoffatoms $\mathrm{H}_{1}$ auf das $\pi$-System ist in den Bindungslängen wie z. B. im 5-Amino1 H-tetrazol [24] und im 2, 4-Dinitrobenzaldehyd (1 Htetrazol-5-yl)hydrazon [25] nicht erkennbar, was gut zu der Tatsache paßt, daß sogar für Tetrazolatanionen ähnliche Abstände gefunden werden, wobei $\mathrm{N}_{2}-\mathrm{N}_{3}$ mit 131 pm etwas länger ist [4 a, 6, 26]. Das $\mathrm{H}_{1}$-Atom an $\mathrm{N}_{1}$ bildet zu $\mathrm{N}_{4}$ eines weiteren Moleküls 8a eine Wasserstoffbrücke $\left(\mathrm{H}_{1}-\mathrm{N}_{4}=195 \mathrm{pm}, \mathrm{N}_{1}-\mathrm{N}_{4}\right.$ $=281 \mathrm{pm}$ ), was zur Ausbildung von Wasserstoff-verbrückten Tetrazol-Ketten im Kristall führt, wie sie ähnlich in 5-Bromtetrazol [23] vorgelegen haben könnten, wobei aber anders als in $\mathbf{8 a}$ das H-Atom nicht lokalisiert werden konnte. 


\begin{tabular}{lllc}
\hline $\mathrm{Cl}(1)-\mathrm{C}(2)$ & $177,5(4)$ & $\mathrm{Cl}(2)-\mathrm{C}(2)$ & $176,6(4)$ \\
$\mathrm{Cl}(3)-\mathrm{C}(2)$ & $176,8(4)$ & $\mathrm{C}(1)-\mathrm{C}(2)$ & $148,6(5)$ \\
$\mathrm{C}(1)-\mathrm{N}(1)$ & $131,8(5)$ & $\mathrm{C}(1)-\mathrm{N}(4)$ & $131,3(5)$ \\
$\mathrm{N}(1)-\mathrm{N}(2)$ & $134,9(4)$ & $\mathrm{N}(2)-\mathrm{N}(3)$ & $127,2(5)$ \\
$\mathrm{N}(3)-\mathrm{N}(4)$ & $137,2(4)$ & $\mathrm{N}(1)-\mathrm{H}(1)$ & $90(4)$ \\
$\mathrm{C}(2)-\mathrm{C}(1)-\mathrm{N}(1)$ & $126,2(3)$ & $\mathrm{C}(2)-\mathrm{C}(1)-\mathrm{N}(4)$ & $125,3(3)$ \\
$\mathrm{N}(1)-\mathrm{C}(1)-\mathrm{N}(4)$ & $108,5(3)$ & $\mathrm{Cl}(1)-\mathrm{C}(2)-\mathrm{Cl}(2)$ & $109,7(2)$ \\
$\mathrm{Cl}(1)-\mathrm{C}(2)-\mathrm{Cl}(3)$ & $108,7(2)$ & $\mathrm{Cl}(2)-\mathrm{C}(2)-\mathrm{Cl}(3)$ & $109,6(2)$ \\
$\mathrm{Cl}(1)-\mathrm{C}(2)-\mathrm{C}(1)$ & $109,4(2)$ & $\mathrm{Cl}(2)-\mathrm{C}(2)-\mathrm{C}(1)$ & $110,4(3)$ \\
$\mathrm{Cl}(3)-\mathrm{C}(2)-\mathrm{C}(1)$ & $109,0(3)$ & $\mathrm{C}(1)-\mathrm{N}(1)-\mathrm{N}(2)$ & $108,8(3)$ \\
$\mathrm{N}(1)-\mathrm{N}(2)-\mathrm{N}(3)$ & $106,7(3)$ & $\mathrm{N}(2)-\mathrm{N}(3)-\mathrm{N}(4)$ & $110,3(3)$ \\
$\mathrm{C}(1)-\mathrm{N}(4)-\mathrm{N}(3)$ & $105,7(3)$ & & \\
\hline
\end{tabular}

Tab. I. Bindungslängen ( $\mathrm{pm})$ und Bindungswinkel (Grad) in $\mathbf{8 a}$.

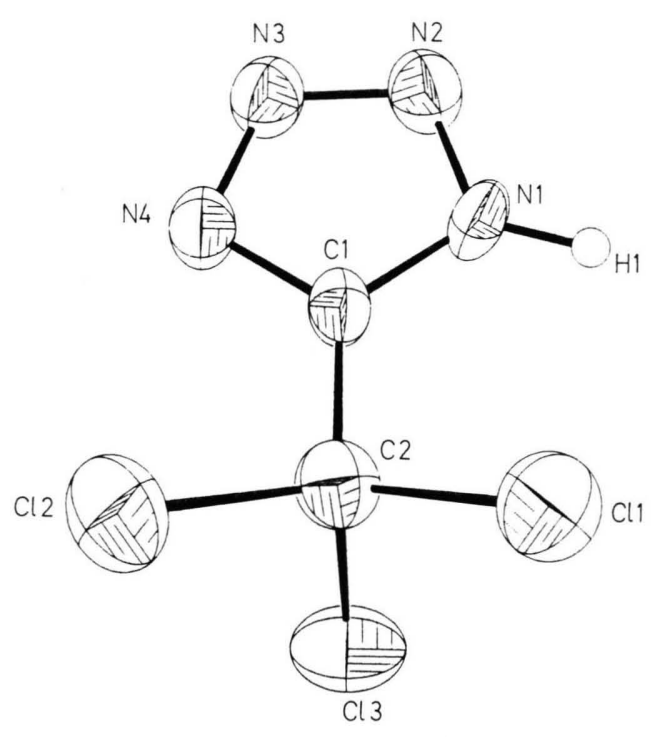

Abb. 1. Molekülstruktur von 8a, die Schwingungsellipsoide entsprechen 50\% Aufenthaltswahrscheinlichkeit, das Wasserstoffatom ist schematisch gezeichnet.

Tab. II. Atomkoordinaten $\left(\times 10^{4}\right)$ und isotrope Temperaturfaktoren $\left(\mathrm{pm}^{2} \cdot 10^{-1}\right)$ in $\mathbf{8 a}$.

\begin{tabular}{lrlll}
\hline Atom & \multicolumn{1}{l}{$x$} & $y$ & $z$ & $\mathrm{U}$ \\
\hline $\mathrm{Cl}(1)$ & $2371(1)$ & $4586(1)$ & $6009(1)$ & $59(1)^{*}$ \\
$\mathrm{Cl}(2)$ & $2309(1)$ & $4518(1)$ & $3033(1)$ & $62(1)^{*}$ \\
$\mathrm{Cl}(3)$ & $688(1)$ & $2152(1)$ & $4330(1)$ & $62(1)^{*}$ \\
$\mathrm{C}(1)$ & $4224(4)$ & $2611(3)$ & $4848(4)$ & $31(1)^{*}$ \\
$\mathrm{C}(2)$ & $2488(4)$ & $3432(4)$ & $4559(4)$ & $40(1)^{*}$ \\
$\mathrm{~N}(1)$ & $5143(4)$ & $2210(3)$ & $6091(4)$ & $37(1)^{*}$ \\
$\mathrm{~N}(2)$ & $6597(4)$ & $1424(3)$ & $5924(3)$ & $44(1)^{*}$ \\
$\mathrm{~N}(3)$ & $6543(4)$ & $1366(3)$ & $4613(3)$ & $43(1)^{*}$ \\
$\mathrm{~N}(4)$ & $5065(4)$ & $2116(3)$ & $3892(3)$ & $39(1)^{*}$ \\
$\mathrm{H}(1)$ & $4848(39)$ & $2638(35)$ & $1922(42)$ & $25(8)$ \\
\hline
\end{tabular}

* Äquivalente isotrope Temperaturfaktoren, definiert als ein Drittel der Spur des orthogonalisierten $\mathrm{U}_{\mathrm{ij}}$-Tensors.

\section{Experimenteller Teil}

Alle Umsetzungen wurden in Schlenkgefäßen unter Stickstoff als Schutzgas durchgeführt. Die Lösungsmittel wurden nach den üblichen Methoden absolutiert.

Die Ausgangskomplexe 1a, b wurden durch Umsetzung der polymeren Schiffbasen mit Co-Acetat und $\mathrm{NaN}_{3}$ erhalten [10].

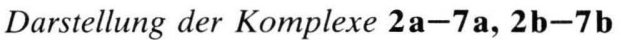 und $10 \mathbf{a}, \mathbf{b}$}

Allgemeine Arbeitsvorschrift: Die Komplexe 1a bzw. 1b werden in $c a$. $60 \mathrm{ml}$ Lösungsmittel suspendiert, mit dem entsprechenden Dipolarophil versetzt und unter Erwärmen gerührt (Stöchiometrie, Reaktionsbedingungen und Reaktionszeit siehe Tab. III). Nach dem Abkühlen zentrifugiert man ab, wäscht dreimal mit je $60 \mathrm{ml} \mathrm{CH}_{2} \mathrm{Cl}_{2}$ und trocknet $10 \mathrm{~h}$ i. Hochvak.

2a: $\mathrm{C}_{19} \mathrm{H}_{14} \mathrm{Cl}_{3} \mathrm{CoN}_{6} \mathrm{O}_{2} \cdot \mathrm{H}_{2} \mathrm{O}(541,7)$

Ber. C 42,13 H 2,98 N 15,52,

Gef. C 42,55 H 3,18 N 14,60.

2b: $\mathrm{C}_{24} \mathrm{H}_{25} \mathrm{Cl}_{3} \mathrm{CoN}_{7} \mathrm{O}_{2}(608,8)$

Ber. C 47,35 H 4,14 N 16,11,

Gef. C 48,06 H 4,78 N 15,00.

7 a: $\mathrm{C}_{19} \mathrm{H}_{19} \mathrm{CoN}_{6} \mathrm{O}_{3}(438,3)$

Ber. C 52,06 H 4,37 N 19,17,

Gef. C 51,37 H 4,39 N 18,29.

Für die übrigen polymeren Komplexe wurden meist zu geringe N-Gehalte gefunden, entsprechend etwa 80-90-proz. Umsatz [27].

5-Trichlormethyltetrazol (8a) aus $\mathbf{2 a}$ bzw. 2 b durch Abspaltung mit $\mathrm{HCl}$

$14,6 \mathrm{~g}(26,9 \mathrm{mmol}) \mathbf{2 a}$ bzw. $12,10 \mathrm{~g}(19,88 \mathrm{mmol})$ 2b werden viermal mit je $40 \mathrm{ml} 0,6 \mathrm{M} \mathrm{HCl} / \mathrm{Et}_{2} \mathrm{O}$ jeweils 15 min verrührt und anschließend dreimal mit je $30 \mathrm{ml} \mathrm{Et}_{2} \mathrm{O}$ gewaschen. Die vereinigten gelben, 
Tab. III. Stöchiometrie, Reaktionsbedingungen zur Darstellung der Verbindungen $2 \mathbf{a}-7 \mathbf{a}, \mathbf{2 b}-7 \mathbf{b}, \mathbf{1 0 a}, \mathbf{b}$.

\begin{tabular}{|c|c|c|c|c|c|}
\hline & Dipolarophil & $\begin{array}{l}\text { 1a: Dipolarophil } \\
(\mathrm{mmol} / \mathrm{mmol})\end{array}$ & $\begin{array}{l}\text { Reaktionszeit } \\
\text { (d) }\end{array}$ & $\begin{array}{l}\text { Reaktionstemp. } \\
\left({ }^{\circ} \mathrm{C}\right)\end{array}$ & $\begin{array}{l}\text { Lösungs- } \\
\text { mittel }\end{array}$ \\
\hline $\mathbf{2 a}$ & Trichloracetonitril & $1: 3,13$ & 14 & R.T. & $\mathrm{CH}_{2} \mathrm{Cl}_{2}$ \\
\hline $2 \mathbf{b}$ & Trichloracetonitril & $1: 3,13$ & 19 & 40 & $\mathrm{CH}_{2} \mathrm{Cl}_{2}$ \\
\hline $3 \mathbf{a}$ & Bernsteinsäuredinitril & $1: 3,13$ & 60 & 65 & $1,2-\mathrm{C}_{2} \mathrm{H}_{4} \mathrm{Cl}$ \\
\hline 3b & Bernsteinsäuredinitril & $1: 4,41$ & 44 & 60 & $\mathrm{CHCl}_{3}$ \\
\hline $4 a$ & Terephthalonitril & $1: 3,13$ & 126 & 60 & $1,2-\mathrm{C}_{2} \mathrm{H}_{4} \mathrm{Cl}$ \\
\hline $4 b$ & Terephthalonitril & $1: 3,13$ & 88 & 60 & $\mathrm{CHCl}_{3}$ \\
\hline $5 \mathbf{a}$ & Isophthalonitril & $1: 3,13$ & 127 & 60 & $1,2-\mathrm{C}_{2} \mathrm{H}_{4} \mathrm{Cl}$ \\
\hline $5 \mathbf{b}$ & Isophthalonitril & $1: 3,13$ & 96 & 60 & $\mathrm{CHCl}_{3}$ \\
\hline $6 a$ & Phthalsäuredinitril & $1: 3,13$ & 132 & 60 & $1,2-\mathrm{C}_{2} \mathrm{H}_{4} \mathrm{Cl}$ \\
\hline $6 \mathrm{~b}$ & Phthalsäuredinitril & $1: 3,13$ & 105 & 45 & Aceton \\
\hline $7 \mathbf{a}$ & Acetonitril & & 60 & Rückfl. & $\mathrm{CH}_{3} \mathrm{CN}$ \\
\hline $7 b$ & Acetonitril & & 38 & 80 & $\mathrm{CH}_{3} \mathrm{CN}$ \\
\hline $10 \mathbf{a}$ & Acetylendicarbonsäuredimethylester & $1: 3,13$ & 14 & R.T. & $\mathrm{CH}_{2} \mathrm{Cl}_{2}$ \\
\hline $10 \mathrm{~b}$ & Acetylendicarbonsäuredimethylester & $1: 3,13$ & 16 & 40 & $\mathrm{CH}_{2} \mathrm{Cl}_{2}$ \\
\hline
\end{tabular}

etherischen Lösungen werden zur Trockene eingeengt und i. Hochvak. bei $80^{\circ} \mathrm{C}$ sublimiert. Das Sublimat wird zweimal aus $\mathrm{CHCl}_{3}$ umkristallisiert. Das farblose kristalline 8a wird bei R.T. 6 h i. Hochvak. getrocknet. Ausbeute: $1,72 \mathrm{~g} \mathrm{(34 \% )}$ sublimiert, $1,06 \mathrm{~g}(21 \%)$ umkristallisiert. Schmp. $127^{\circ} \mathrm{C}$ (unter $\mathrm{N}_{2}$-Entwicklung). IR $\left(\mathrm{KBr}, \mathrm{cm}^{-1}\right): 3080 \mathrm{~m} \mathrm{(NH)}$, 2966-2439 m (NH-Brücken), $1549 \mathrm{~s}, 1451 \mathrm{ww}$, $1423 \mathrm{~s}, 1374 \mathrm{~s}, 1237 \mathrm{~s}, 1119 \mathrm{vs}, 1101 \mathrm{~s}, 1091 \mathrm{~s}$, $1042 \mathrm{ss}, 1008 \mathrm{~m}, 1000 \mathrm{~s}, 935 \mathrm{~m}, \mathrm{br}, 845 \mathrm{~s}, 836 \mathrm{~s}$, 805 vs, 792 s, 723 vs, 701 vs, $390 \mathrm{~m}, 387 \mathrm{w}, 383 \mathrm{w}$. MS $\left(70 \mathrm{eV}, 65^{\circ} \mathrm{C}\right): m / e=192\left(2 \%, \mathrm{M}^{+}\right), 190(6 \%$, $\left.\mathrm{M}^{+}\right), 188\left(4 \%, \mathrm{M}^{+}\right), 186\left(3 \%, \mathrm{M}^{+}\right), 127(38 \%), 125$ $(100 \%), 123\left(100 \%, \mathrm{M}^{+}-\mathrm{N}_{2}-\mathrm{Cl}\right.$ oder $\left.-\mathrm{C}^{37} \mathrm{Cl}_{3}\right), 69$ $\left(58 \%, \mathrm{CN}_{4} \mathrm{H}\right)$ (ausgewählte Werte).

$$
\begin{array}{rrrr}
\mathrm{C}_{2} \mathrm{HCl}_{3} \mathrm{~N}_{4} & (187,4) \\
\text { Ber. } & \mathrm{C} 12,82 & \text { H 0,54 } & \text { N 29,89, } \\
\text { Gef. } & \text { C } 12,90 & \text { H } 0,21 & \text { N 29,85. }
\end{array}
$$

Die Abspaltung von 8a kann auch mit $\mathrm{HCl} /$ $\mathrm{CH}_{3} \mathrm{OH}$ vorgenommen werden. Dabei sinken die Ausbeuten auf unter $1 \%$.

Regenerierung der Azido-Komplexe 1 und erneute Abspaltung von Heterocyclen

Der bei der Darstellung von 8a aus 2a angefallene polymere Chloro-Komplex wird i. Hochvak. getrocknet.

$$
\begin{array}{rlll}
\mathrm{C}_{17} \mathrm{H}_{16} \mathrm{ClCoN}_{2} \mathrm{O}_{3}(390,7) & \\
\text { Ber. } & \mathrm{C} 52,26 & \mathrm{H} 4,13 & \text { N 7,17, } \\
\text { Gef. } & \text { C } 50,71 & \mathrm{H} 4,47 & \text { N 8,74. }
\end{array}
$$

$10 \mathrm{~g}$ des polymeren Chlorocobalt-Komplexes werden $6 \mathrm{~h}$ bei $20^{\circ} \mathrm{C}$ in $100 \mathrm{ml}$ einer mit $\mathrm{NaN}_{3}$ gesättigten Lösung aus $\mathrm{H}_{2} \mathrm{O} / \mathrm{C}_{2} \mathrm{H}_{5} \mathrm{OH}$ (1:1) gerührt. Nach dem Abfiltrieren und Waschen mit Wasser und
Ethanol wird der Feststoff $10 \mathrm{~h}$ i. Hochvak. getrocknet.

Nach einer weiteren Cycloaddition und Abspaltung wird 8a in $9 \%$ Ausbeute isoliert. Ebenso wurde mit dem Trägerkomplex aus der Darstellung von 8a über $\mathbf{2 b}$ verfahren. Der Zyklus wurde hier zweimal wiederholt und führte zu Ausbeuten von $0,61 \mathrm{~g}$ $(17 \%)$ und $0,45 \mathrm{~g}(13 \%) \mathbf{8 a}$.

\section{3-(5-Tetrazolyl)-propionamid (8b) aus $\mathbf{3 a}$ durch Abspaltung mit $\mathrm{HCl}$}

Ein 1-l-Schlenkkolben mit $14,25 \mathrm{~g}(29,85 \mathrm{mmol})$ 3a wird evakuiert und mit $\mathrm{HCl}-\mathrm{Gas}$ versetzt. Es erfolgt ein langsamer Farbumschlag von braun nach dunkelgrün. Nach 30 min wird weiteres $\mathrm{HCl}-\mathrm{Gas}$ in den Kolben geleitet, um den entstandenen Unterdruck auszugleichen. Nun läßt man $46 \mathrm{~h}$ bei gelegentlichem Umschütteln stehen. Nach dem Absaugen des $\mathrm{HCl}-\mathrm{G}$ ases wird der Feststoff dreimal mit je $25 \mathrm{ml}$ Aceton gewaschen. Die gesammelten Acetonlösungen werden eingeengt und der daraus erhaltene grüne Rückstand wird mit $\mathrm{CH}_{2} \mathrm{Cl}_{2}$ gewaschen und anschließend mehrmals aus Aceton umkristallisiert. Der blaßgelbe Feststoff wird nun $6 \mathrm{~h}$ i. Hochvak. getrocknet. Ausbeute: $12 \mathrm{mg}(0,3 \%)$. IR $\left(\mathrm{KBr}, \mathrm{cm}^{-1}\right): 3368 \mathrm{ss}$, 3320 sh, 3197 ss $\left(\mathrm{NH}_{2}\right), 3100-2600$ (H-Brücken), 1673 ss $(\mathrm{C}=\mathrm{O}), 1629 \mathrm{~s}\left(\mathrm{NH}_{2}\right), 1547 \mathrm{~s}, 1421 \mathrm{~s}, 1403 \mathrm{~s}$, $1248 \mathrm{~m}, 1217 \mathrm{~m}, 1079 \mathrm{~m}, 878 \mathrm{~m}$. MS $\left(70 \mathrm{eV}, 110{ }^{\circ} \mathrm{C}\right)$ : $m / e=141\left(2 \%, \mathrm{M}^{+}\right), 125\left(5 \%, \mathrm{M}^{+}-\mathrm{NH}_{2}\right), 113(4 \%$, $\left.\mathrm{M}^{+}-\mathrm{N}_{2}\right), 99\left(59 \%, \mathbf{M}^{+}-\mathrm{N}_{3}\right), 98\left(20 \%, \mathbf{M}^{+}-\mathrm{HN}_{3}\right), 84$ $\left(15 \%, \mathrm{M}^{+}-\mathrm{N}_{4} \mathrm{H}\right), 72\left(100 \%, \mathrm{CH}_{2} \mathrm{CH}_{2} \mathrm{CONH}_{2}{ }^{+}\right), 69$ $\left(28 \%, \mathrm{HN}_{4} \mathrm{C}^{+}\right)$.

$$
\begin{aligned}
& \mathrm{C}_{4} \mathrm{H}_{7} \mathrm{~N}_{5} \mathrm{O}(141,1) \\
& \text { Ber. C 34,04 H 5,00 N 49,62, } \\
& \text { Gef. C 34,85 H 5,52 N 46,79. }
\end{aligned}
$$


5-(Cyanophenyl)tetrazole $(\mathbf{8 c}, \mathbf{d}, \mathbf{e})$ aus $(\mathbf{4 a}, \mathbf{5 a}, \mathbf{6 a})$ durch Abspaltung mit $\mathrm{HCl}$

$13,0 \mathrm{~g}(24,7 \mathrm{mmol}) \mathbf{4 a}(13,0 \mathrm{~g}(24,7 \mathrm{mmol}) \mathbf{5 a}$; $13,6 \mathrm{~g}(25,9 \mathrm{mmol}) \mathbf{6 a})$ werden in $60 \mathrm{ml} \mathrm{Et}_{2} \mathrm{O}$ und $5 \mathrm{ml}$ konz. $\mathrm{HCl}$ suspendiert und $16 \mathrm{~h}$ gerührt (6a: $\left.100 \mathrm{ml} \mathrm{Et}_{2} \mathrm{O}, 5 \mathrm{ml} \mathrm{HCl}, 15 \mathrm{~h}\right)$. Der Feststoff wird abgefrittet und dreimal mit $40 \mathrm{ml} \mathrm{Et}_{2} \mathrm{O}$ (6a: zweimal mit $80 \mathrm{ml} \mathrm{Et}_{2} \mathrm{O}$ ) gewaschen. Die vereinigten etherischen Lösungen werden zur Trockene eingeengt. Der Rückstand wird in $20 \mathrm{ml} \mathrm{Et}_{2} \mathrm{O}$ aufgenommen und zweimal mit je $20 \mathrm{ml} \mathrm{H}_{2} \mathrm{O}$ ausgeschüttelt (8e $60 \mathrm{ml} \mathrm{Et}_{2} \mathrm{O}, 30 \mathrm{ml} \mathrm{H}_{2} \mathrm{O}$ ).

$8 \mathbf{c}$ erhält man durch Einengen der $\mathrm{Et}_{2} \mathrm{O}$-Phase, umkristallisieren aus EtOH und Trocknen i. Hochvak. Ausbeute: $12 \mathrm{mg}(0,24 \%)$. Die etherischen Lösungen von $\mathbf{8 d}$ und $\mathbf{8 e}$ werden zur Trockene eingeengt, der Rückstand mit $10 \mathrm{ml}$ (8e: $20 \mathrm{ml}) 1 \mathrm{~N}$ $\mathrm{NaOH}$ gelöst, abfiltriert und wieder mit $6 \mathrm{~N} \mathrm{HCl}$ angesäuert. Das ausgefallene Produkt wird mehrmals aus EtOH umkristallisiert und i. Hochvak. getrocknet. Ausbeute: 8d: $11 \mathrm{mg}(0,3 \%), \mathbf{8 e}: 10 \mathrm{mg}$ $(0,2 \%)$. IR $\left(\mathrm{KBr}, \quad \mathrm{cm}^{-1}\right): \mathbf{8 c}: 3098 \mathrm{~s} \quad(\mathrm{NH})$, 3000-2400 s (NH-Brücken), $2238 \mathrm{~m}(\mathrm{CN}), 1575 \mathrm{ss,}$ $1561 \mathrm{~s}, 1499 \mathrm{ss}, 1435 \mathrm{ss}, 1218 \mathrm{~s}, 1152 \mathrm{~s}, 1059 \mathrm{~s}$, 992 ss, 850 ss, 751 s, 701 s, 555 s. 8d: 3095 m (NH), $3000-2480 \mathrm{~s}(\mathrm{NH}), 2235 \mathrm{~m}(\mathrm{CN}), 1612 \mathrm{~m}, 1561 \mathrm{~s}$, $1481 \mathrm{~s}, 1445 \mathrm{~m}, 1425 \mathrm{~m}, 1249 \mathrm{~m}, 1202 \mathrm{~m}, 1090 \mathrm{~s}$, 1061 s, 1030 s, 918 s, 800 s, 744 ss. 8e: $3080-2420$ m (NH-Brücken), $2232 \mathrm{~m} \mathrm{(CN),} 1609 \mathrm{~m}, 1581 \mathrm{~m}$, $1492 \mathrm{~m}, 1453 \mathrm{~m}, 1408 \mathrm{~m}, 1240 \mathrm{~m}, 1167 \mathrm{~s}, 1065 \mathrm{~s}$, $1048 \mathrm{~m}, 1012 \mathrm{~m}, 758 \mathrm{ss}$.

\begin{tabular}{|c|c|c|c|}
\hline \multicolumn{4}{|c|}{ 8c: $\mathrm{C}_{8} \mathrm{H}_{5} \mathrm{~N}_{5} \cdot \mathrm{H}_{2} \mathrm{O}(198,2)$} \\
\hline Ber. & C 50,79 & H 3,73 & N 37,02 , \\
\hline Gef. & C 52,22 & H 3,74 & N 37,05 . \\
\hline \multicolumn{4}{|c|}{ 8d: $\mathrm{C}_{8} \mathrm{H}_{5} \mathrm{~N}_{5}(171,2)$} \\
\hline Ber. & C 56,14 & H 2,94 & N 40,92, \\
\hline Gef. & C 59,86 & Н 3,60 & N 39,65 . \\
\hline \multicolumn{4}{|c|}{ 8e: $\mathrm{C}_{8} \mathrm{H}_{5} \mathrm{~N}_{5}(171,2)$} \\
\hline Ber. & C 56,14 & H 2,94 & $\mathrm{~N} 40,9$ \\
\hline Gef. & C 56,32 & H 3,25 & N 40,6 \\
\hline
\end{tabular}

\section{5-Methyltetrazol 8f aus 7a durch Abspaltung mit $\mathrm{HCl}$}

$11,0 \mathrm{~g}(25,07 \mathrm{mmol}) \mathbf{7 a}$ werden zweimal in je $35 \mathrm{ml} \mathrm{CH} \mathrm{CH}_{3} \mathrm{OH} / \mathrm{HCl} 30 \mathrm{~min}$ suspendiert. AnschlieBend wird der Feststoff zweimal mit je $30 \mathrm{ml}$ Aceton gewaschen und die vereinigten Lösungen zur Trokkene eingeengt. Der verbleibende grüne Rückstand wird zweimal mit je $25 \mathrm{ml}$ Methylenchlorid bei R.T. gewaschen. Aus dem Rückstand läßt sich $8 \mathbf{f}$ mit heiBem Chloroform (fünfmal mit je $20 \mathrm{ml}$ ) eluieren. Nach dem Einengen der gelben Chloroformlösungen auf $25 \mathrm{ml}$ und Abkühlen im Eisbad kann man das auskristallisierte, auf der Oberfläche schwimmende 8f abfiltrieren. Umkristallisieren aus Chloroform und Trocknen i. Hochvak. liefert das farblose kristalline 8f. Ausbeute $0,35 \mathrm{~g}(17 \%)$. IR $\left(\mathrm{KBr}, \mathrm{cm}^{-1}\right)$ : 3141-2350 s (NH-Brücken), 1580 s, 1561 s, $1266 \mathrm{~m}$, $1254 \mathrm{~s}, 1110 \mathrm{ss}, 1050 \mathrm{ss}, 680 \mathrm{~s}$.

$\mathrm{C}_{2} \mathrm{H}_{4} \mathrm{~N}_{4}(84,1)$

Ber. C 28,57 H 4,79 N 66,63,

Gef. C 28,80 H 4,82 N 66,65.

$\mathrm{N}$-Acetyl-5-trichlormethyl-tetrazol (9) durch Reaktion von $\mathbf{2 a}$ mit Acetylchlorid

$14,50 \mathrm{~g}(26,77 \mathrm{mmol}) \mathbf{2 a}$ werden in $10 \mathrm{ml}$ Acetylchlorid und $20 \mathrm{ml} \mathrm{Et}_{2} \mathrm{O} 30 \mathrm{~min}$ suspendiert. Dieser Vorgang wird noch zweimal wiederholt, die Lösungen werden vereinigt und eingeengt. Durch Zugabe von $30 \mathrm{ml}$ Pentan erhält man einen festen Rückstand, der aus Trichloracetamid besteht. Die abgetrennte Pentan-Lösung wird eingeengt und das gelbe Öl i. Hochvak. getrocknet. IR $\left(\mathrm{KBr}, \mathrm{cm}^{-1}\right)$ : $1800(\mathrm{C}=\mathrm{O}) .{ }^{1} \mathrm{H}-\mathrm{NMR}\left(\mathrm{CD}_{2} \mathrm{Cl}_{2}\right): \delta=4,47,4,37$ (N(1)-Isomeres), 3,03 (N(2)-Isomeres); Intensitätsverhältnisse $5: 1: 6$; die beiden Signale für das N(1)Isomere haben ihre Ursache in der eingeschränkten Rotation der Acetylgruppe. Zum Vergleich wurde 9 direkt durch Umsetzung von 8a mit Acetylchlorid dargestellt; $0,37 \mathrm{~g}(2 \mathrm{mmol}) \mathbf{8 a}$ in Acetylchlorid $4 \mathrm{~h}$ rückflußkochen. Schmp. $58-62{ }^{\circ} \mathrm{C}$.

$$
\begin{array}{rrrr}
\mathrm{C}_{4} \mathrm{H}_{3} \mathrm{Cl}_{3} \mathrm{~N}_{4} \mathrm{O} & (229,4) \\
\text { Ber. } & \mathrm{C} 20,94 & \mathrm{H} 1,32 & \mathrm{~N} 24,42, \\
\text { Gef. } & \mathrm{C} 22,57 & \mathrm{H} 1,55 & \mathrm{~N} 23,20 .
\end{array}
$$

${ }^{1} \mathrm{H}$-NMR $\left(\mathrm{CDCl}_{2}\right): \delta=3,00(\mathrm{~N}(2)$-Isomeres). Hydrolyse von 9 ( 15 h Rühren in $\mathrm{CH}_{2} \mathrm{Cl}_{2} / \mathrm{H}_{2} \mathrm{O}$ bei $20{ }^{\circ} \mathrm{C}$ ) ergibt wieder $\mathbf{8 a}$.

\section{4,5-Di(methoxycarbonyl)-triazol (11) aus 10a bzw. $10 \mathbf{b}$ durch Abspaltung mit $\mathrm{HCl}$}

$13,0 \mathrm{~g}(24,1 \mathrm{mmol}) \mathbf{1 0 a}(16,76 \mathrm{~g}(27,63 \mathrm{mmol})$ 10 b) werden viermal mit je $40 \mathrm{ml} \mathrm{Et}_{2} \mathrm{O} / \mathrm{HCl}(0,6 \mathrm{~N}$ an $\mathrm{HCl}$ ) jeweils $15 \mathrm{~min}$ verrührt. Dabei erfolgt ein Farbumschlag von braun nach dunkelgrün. AnschlieBend wird dreimal mit je $30 \mathrm{ml} \mathrm{Et}_{2} \mathrm{O}$ gewaschen. Die vereinigten Etherphasen werden zur Trockene eingeengt und der gelb-grüne Rückstand wird i. Hochvak. bei $100{ }^{\circ} \mathrm{C}$ sublimiert. Das hellgelbe Sublimat wird zweimal aus heißem $\mathrm{CCl}_{4}$ umkristallisiert und der farblose kristalline Feststoff $\mathbf{1 1}$ sechs Stunden bei R.T. i. Hochvak. getrocknet. Ausbeute (sublimiert): $1,56 \mathrm{~g} \quad(35 \%)$. Ausb. (umkristallisiert): $1,10 \mathrm{~g}$ $(24,6 \%)$, Schmp. $126-131{ }^{\circ} \mathrm{C}$ (Zers.). IR ( $\mathrm{KBr}$, 
$\mathrm{cm}^{-1}$ ): $3240 \mathrm{ss}, 2960 \mathrm{~m}, 1743 \mathrm{ss}, \mathrm{br}, 1521 \mathrm{~m}, 1388 \mathrm{~s}$, 1301 s, 1190 m, 1081 ss, 990 m, 836 m, 772 s.

$\begin{array}{rrrr}\mathrm{C}_{6} \mathrm{H}_{7} \mathrm{~N}_{3} \mathrm{O}_{4} & (185,1) \\ \text { Ber. } & \text { C } 38,93 & \text { H 3,81 } & \text { N 22,70, } \\ \text { Gef. } & \text { C } 38,78 & \text { H 3,96 } & \text { N 22,71. }\end{array}$

(Tetraphenylporphinato)cobalt(III)-tetrafluoroborat (12) und

Azido(tetraphenylporphinato)-cobalt(III) (13)

$490 \mathrm{mg}(0,72 \mathrm{mmol}) \mathrm{Co}\left(\mathrm{CH}_{3}\right)$ (TPP) [19] werden in $30 \mathrm{ml} \mathrm{CH}_{2} \mathrm{Cl}_{2}$ mit $0,099 \mathrm{ml} \mathrm{HBF}_{4}$-Etherat versetzt. Nach 3 h Rühren bei $20{ }^{\circ} \mathrm{C}$ wird durch langsame Zugabe von $50 \mathrm{ml} n$-Pentan das grauviolette Produkt ausgefällt. Es wird abzentrifugiert, dreimal mit je $10 \mathrm{ml} n$-Pentan gewaschen und $4 \mathrm{~h}$ i. Hochvak. getrocknet. Ausbeute: $110 \mathrm{mg}$ (20\%). IR (Nujol, $\left.\mathrm{cm}^{-1}\right): 1050 \mathrm{~s}\left(\mathrm{BF}_{4}\right) .{ }^{1} \mathrm{H}-\mathrm{NMR}\left(\mathrm{CD}_{2} \mathrm{Cl}_{2}\right): \delta=8,89$, $8,41,7,78$ (TPP).

\section{$\mathrm{C}_{44} \mathrm{H}_{28} \mathrm{BCoF}_{4} \mathrm{~N}_{4}(758,5)$ \\ Ber. $\quad \mathrm{N} 7,39$, \\ Gef. $\quad$ 7 7,31.}

$60 \mathrm{mg}(0,08 \mathrm{mmol}) 12$ werden mit $52 \mathrm{mg} \mathrm{\textrm {NaN } _ { 3 }}$ $(0,8 \mathrm{mmol})$ in $10 \mathrm{ml} \mathrm{CH} \mathrm{Cl}_{2} 18 \mathrm{~h}$ bei R.T. gerührt. Der entstandene $\mathrm{NaBF}_{4}-\mathrm{Niederschlag}$ wird abzentrifugiert und das Produkt als braunvioletter Niederschlag durch Zugabe von $15 \mathrm{ml}$ Pentan zum Filtrat ausgefällt. Es wird abzentrifugiert, dreimal mit je $5 \mathrm{ml} n$-Pentan gewaschen und $5 \mathrm{~h}$ i. Hochvak. getrocknet. IR (Nujol, $\left.\mathrm{cm}^{-1}\right): 2000$ vs $\left(\nu_{\mathrm{as}} \mathrm{N}_{3}\right), 1265 \mathrm{w}$ $\left(v_{\mathrm{s}} \mathrm{N}_{3}\right)$. Ausbeute: $45 \mathrm{mg}(81 \%)$.

$\begin{array}{rrrr}\mathrm{C}_{44} \mathrm{H}_{28} \mathrm{CoN}_{7}(713,69) & & \\ \text { Ber. } & \mathrm{C} 74,05 & \text { H 3,95 } & \text { N 13,74, } \\ \text { Gef. } & \text { C 71,88 } & \text { H 3,52 } & \text { N 12,78. }\end{array}$

Triazolato(tetraphenylporphinato)-cobalt(III) und Abspaltung von 4,5-Di(methoxycarbonyl)-triazol (11)

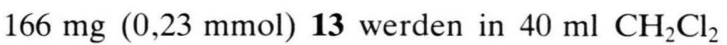

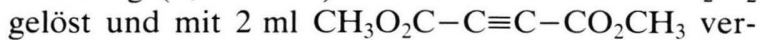
setzt. Nach 2 d Rühren bei R.T. wird der Triazolatkomplex durch Fällen mit $60 \mathrm{ml} n$-Pentan erhalten. Es wird mit viel $n$-Pentan gewaschen und $3 \mathrm{~h}$ i. Hochvak. getrocknet. Ausbeute: $124 \mathrm{mg}$ (65\%). $70 \mathrm{mg}(0,08 \mathrm{mmol})$ dieser Verbindung werden in $20 \mathrm{ml} \mathrm{EtOH}$ und $1 \mathrm{ml}$ verd. $\mathrm{HCl}$ suspendiert. Der rotviolette Niederschlag ( $\mathrm{CoCl}(\mathrm{TPP})$ ) wird abzentrifugiert, zweimal mit EtOH gewaschen und i. Hochvak. getrocknet. Die EtOH-Lösungen werden eingeengt und das verbliebene Öl sublimiert. Das abgeschiedene Triazol (11) und der Co-Komplex werden IR-spektroskopisch identifiziert.
(Tetraphenylporphinato) tricyanmethanidocobalt (III) (15)

Eine Lösung von $100 \mathrm{mg}(0,13 \mathrm{mmol}) \mathbf{1 2}$ und $17 \mathrm{mg} \mathrm{KC}(\mathrm{CN})_{3}(0,13 \mathrm{mmol})$ wird $20 \mathrm{~h}$ bei R.T. gerührt. Der entstandene $\mathrm{KBF}_{4}$-Niederschlag wird abzentrifugiert und das Filtrat mit $20 \mathrm{ml} n$-Pentan versetzt. Der ausgefallene Niederschlag wird dreimal mit je $5 \mathrm{ml} n$-Pentan gewaschen und $4 \mathrm{~h}$ i. Hochvak. getrocknet. Ausbeute: $73 \mathrm{mg}$ (73\%). IR (Nujol, $\left.\mathrm{cm}^{-1}\right): 2165 \mathrm{~s}, 2140 \mathrm{~s}(\mathrm{CN})$.

$\mathrm{C}_{48} \mathrm{H}_{28} \mathrm{CoN}_{7}(761,73)$

Ber. C 75,69 H 3,71 N 12,87,

Gef. C 76,53 H 4,40 N 12,81.

Reaktion von Azido-(tetraphenylporphinato)cobalt(III) mit Cyclohexylisonitril (14)

Eine Lösung von $118 \mathrm{mg}(0,17 \mathrm{mmol}) \mathbf{1 3}$ und $1 \mathrm{ml}$

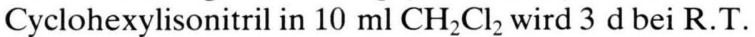
gerührt. Dann wird das grüne Produkt mit $20 \mathrm{ml}$ $n$-Pentan ausgefällt, abzentrifugiert, dreimal mit je $5 \mathrm{ml} n$-Pentan gewaschen und i. Hochvak. getrocknet. Ausbeute: $83 \mathrm{mg}(61 \%)$. IR (Nujol, $\left.\mathrm{cm}^{-1}\right)$ : $2930 \mathrm{~s}, 2854 \mathrm{~s}, 1461 \mathrm{~m}, 1449 \mathrm{~m}(\mathrm{CH}) .{ }^{1} \mathrm{H}-\mathrm{NMR}$ $\left(\mathrm{CD}_{2} \mathrm{Cl}_{2}\right): \delta=7,84$ (TPP), 1,36 (CH).

$\mathrm{C}_{51} \mathrm{H}_{41} \mathrm{CoN}_{8} \mathrm{O}(840,88)$

Ber. C 72,85 H 4,91 N 13,33,

Gef. C 70,31 H 4,92 N 14,11.

Umsetzung von (TPP) $\mathrm{CoN}_{3} \cdot \mathrm{H}_{2} \mathrm{O}$ mit

a) Trichloracetonitril, b) Acetonitril und

c) Acetylendicarbonsäuredimethylester und

Abspaltung der Tetrazole bzw. Triazole

a) $0,55 \mathrm{~g}(0,75 \mathrm{mmol})(\mathrm{TPP}) \mathrm{CoN}_{3} \cdot \mathrm{H}_{2} \mathrm{O}$ werden mit $0,7 \mathrm{ml}(6,8 \mathrm{mmol}) \mathrm{Cl}_{3} \mathrm{CCN}$ in $10 \mathrm{ml} \mathrm{CH}_{2} \mathrm{Cl}_{2} 7 \mathrm{~d}$ bei R.T. gerührt. Dann wird zur Trockene eingeengt und i. Hochvak. getrocknet.

$$
\begin{aligned}
& \mathrm{C}_{46} \mathrm{H}_{30} \mathrm{Cl}_{3} \mathrm{CoN}_{8} \mathrm{O}(876,1) \\
& \text { Ber. } \quad \mathrm{N} 12,79 \text {, } \\
& \text { Gef. } \quad \mathrm{N} 12,16 \text {. }
\end{aligned}
$$

b) $0,50 \mathrm{~g}(0,68 \mathrm{mmol})(\mathrm{TPP}) \mathrm{CoN}_{3} \cdot \mathrm{H}_{2} \mathrm{O}$ werden $7 \mathrm{~d}$ in $20 \mathrm{ml}$ Acetonitril rückflußgekocht. Es wird zur Trockene eingeengt und i. Hochvak. getrocknet.

$$
\begin{array}{rlll}
\mathrm{C}_{46} \mathrm{H}_{33} \mathrm{CoN}_{8} \mathrm{O}(772,7) & & \\
\text { Ber. } & \mathrm{C} 71,50 & \mathrm{H} 4,30 & \text { N 14,50, } \\
\text { Gef. } & \text { C 74,55 } & \text { H 4,33 } & \text { N 12,37. }
\end{array}
$$

c) $166 \mathrm{mg}(0,23 \mathrm{mmol})(\mathrm{TPP}) \mathrm{CoN}_{3} \cdot \mathrm{H}_{2} \mathrm{O}$ und $2 \mathrm{ml}$ $\mathrm{CH}_{3} \mathrm{O}_{2} \mathrm{C}-\mathrm{C} \equiv \mathrm{C}-\mathrm{CO}_{2} \mathrm{CH}_{3}$ werden in $40 \mathrm{ml} \mathrm{CH}_{2} \mathrm{Cl}_{2}$ $2 \mathrm{~d}$ bei R.T. gerührt. Das Produkt wird mit $n$-Pentan 
ausgefällt, gewaschen und i. Hochvak. getrocknet. Ausbeute: $124 \mathrm{mg}(65 \%)$

$\mathrm{C}_{50} \mathrm{H}_{38} \mathrm{CoN}_{7} \mathrm{O}_{5}(873,8)$
Ber.
Gef. 68,73 H 4,38,
Gef 69,11 H 4,77.

\section{Abspaltung der Heterocyclen $\mathbf{8 a}, \mathbf{8 f}$ und $\mathbf{1 1}$}

Allg. Arbeitsvorschrift: ca. $0,1 \mathrm{mmol}$ der Komplexe werden in $20 \mathrm{ml} \mathrm{EtOH}$ mit $1 \mathrm{ml}$ verd. $\mathrm{HCl} 1 \mathrm{~h}$ bei R.T. gerührt. Man zentrifugiert ab und wäscht zweimal mit EtOH und sublimiert. Die Produkte werden IR-spektroskopisch identifiziert.

\section{Reaktionen mit polymeren Diazido-bis(phosphan)- palladium(II)-Komplexen}

Darstellung von polymerem Diazido-bis(phosphan)palladium(II) (16)

a) Polymeres Benzyldiphenylphosphan $[28,29]$

$18,8 \mathrm{~g}(71,7 \mathrm{mmol})$ Triphenylphosphan und 7,31 g $(187 \mathrm{mmol})$ Kalium werden in $150 \mathrm{ml}$ Dioxan $5 \mathrm{~h}$ zum Sieden erhitzt. Man läßt auf $60^{\circ} \mathrm{C}$ abkühlen und filtriert bei 270 mbar durch eine mit siedendem $\mathrm{Di}$ oxan beheizte Fritte, die $5 \mathrm{~mm}$ hoch mit geglühtem Kieselgur bedeckt ist. Dem Filtrat werden $20 \mathrm{ml}$ Toluol zugegeben, worauf beim Abkühlen $23,3 \mathrm{~g}$ $(58,2 \mathrm{mmol}) \mathrm{KPPh}_{2} \cdot 2$ Dioxan auskristallisieren, die abfiltriert und i. Hochvak. getrocknet werden.

$10 \mathrm{~g}(25 \mathrm{mmol}) \mathrm{KPPh}_{2} \cdot 2$ Dioxan werden in $100 \mathrm{ml}$ THF gelöst und $17 \mathrm{~g}$ (24 mval) sorgfältig von Luft befreitem Merrifield-Harz (bezogen von Fa. Merck, Darmstadt, chlormethyliertes mit 2\% Divinylbenzol vernetztes Polystyrol; 5,8\% Cl) zugegeben. Nach einstündigem Rühren bei R.T. wird das Polymere abfiltriert, mit viel Wasser, Ethanol und Ether gründlich gewaschen und i. Hochvak. getrocknet.

$\left[\left(\mathrm{C}_{42.4} \mathrm{H}_{42.3} \mathrm{Cl}_{0.13}\right)-\mathrm{C}_{9} \mathrm{H}_{9}-\mathrm{PPh}_{2}\right]_{n}$ (1 mol Phosphan entspricht etwa $859 \mathrm{~g}$ )

Ber. C 88,66 H 7,19 P 3,61,

Gef. C 89,07 H 7,38 P 3,49.

b) Umsetzung von polymerem Phosphan mit Natriumtetrachloropalladat

$400 \mathrm{mg}(1,36 \mathrm{mmol}) \mathrm{Na}_{2} \mathrm{PdCl}_{4}$ werden in $15 \mathrm{ml}$ $\mathrm{H}_{2} \mathrm{O}$ gelöst, $5 \mathrm{ml}$ Ethanol und $400 \mathrm{mg}(0,47 \mathrm{mval})$ polymeres Phosphan zugegeben und $12 \mathrm{~h}$ gerührt. Danach wird das gelbe Polymere abfiltriert, mit $\mathrm{H}_{2} \mathrm{O}$, EtOH und $\mathrm{Et}_{2} \mathrm{O}$ gründlich gewaschen und i. Hochvak. getrocknet.

$\left[\mathrm{C}_{63.4} \mathrm{H}_{61.3} \mathrm{Cl}_{0.13} \mathrm{P}\right]_{n}\left[\mathrm{PdCl}_{2}\right]_{0.28 n / 2}$

Beladung: Atomverhältnis $\mathrm{P}: \mathrm{Pd}=7,2: 1$.
(Ermittelt aus dem C:N-Verhältnis nach Austausch der Chloro- gegen Azidoliganden.)

c) Umsetzung von polymerem Phosphan mit Dichlorobis(triphenylphosphan)palladium

Die eintägige Reaktion einer Lösung von $500 \mathrm{mg}$ $(0,71 \mathrm{mmol}) \quad\left(\mathrm{Ph}_{3} \mathrm{P}\right)_{2} \mathrm{PdCl}_{2}$ in $20 \mathrm{ml} \mathrm{CH}_{2} \mathrm{Cl}_{2}$ mit $200 \mathrm{mg}(0,23 \mathrm{mval})$ polymerem Phosphan führt nach Filtrieren, Waschen mit $\mathrm{CH}_{2} \mathrm{Cl}_{2}$ und $\mathrm{Et}_{2} \mathrm{O}$ und Trocknen i. Hochvak. zum gelben polymeren $\mathrm{Di}$ chlorophosphanpalladium.

$\left[\mathrm{C}_{63.4} \mathrm{H}_{61.3} \mathrm{Cl}_{0.13} \mathrm{P}\right]_{n}\left[\mathrm{PdCl}_{2}\right]_{0.42 n / 2}$

Beladung: Atomverhältnis $\mathrm{P}: \mathrm{Pd}=4,8: 1$.

$5 \mathrm{~g}$ [Mer- $\left.\mathrm{PPh}_{2}\right]_{2}\left[\mathrm{PdCl}_{2}\right]_{x}$, dargestellt nach b) werden mit $10 \mathrm{~g} \mathrm{NaN}_{3}$ in feuchtem THF gerührt, nach $2 \mathrm{~d}$ abfiltriert, mit THF, $\mathrm{H}_{2} \mathrm{O}$, EtOH und $\mathrm{Et}_{2} \mathrm{O}$ gewaschen und das Produkt 16a i. Hochvak. getrocknet. IR $(\mathrm{KBr}): 2023 \mathrm{~s}\left(v_{\mathrm{as}} \mathrm{N}_{3}\right), 1267 \mathrm{w}\left(v_{\mathrm{s}} \mathrm{N}_{3}\right)$.

$\left[\mathrm{C}_{63.4} \mathrm{H}_{61.3} \mathrm{Cl}_{0.13} \mathrm{P}\right]_{n}\left[\mathrm{Pd}\left(\mathrm{N}_{3}\right)_{2}\right]_{0.28 n / 2}$

Ber. C 85,99 H 6,98 N 1,33 Pd 1,68,

Gef. C 86,43 H 7,61 N 1,33 Pd 1,48.

Die analoge Umsetzung mit dem nach c) dargestellten polymeren Phosphankomplex ergibt 16b:

$\left[\mathrm{C}_{63.4} \mathrm{H}_{61.3} \mathrm{Cl}_{0.13} \mathrm{P}\right]_{n}\left[\mathrm{Pd}\left(\mathrm{N}_{3}\right)_{2}\right]_{0.42 n / 2}$

Ber. C 84,72 H 6,87 N 1,96,

Gef. C 85,39 H 7,40 N 1,96.

Polymere Diphosphan-bis(5-R-tetrazolato)palladium(II)-Komplexe $(\mathbf{1 7} \mathbf{a}-\mathbf{e})$

$400 \mathrm{mg}\left[\mathrm{Mer}-\mathrm{PPh}_{2}\right]_{2}\left[\mathrm{Pd}\left(\mathrm{N}_{3}\right)_{2}\right]_{0.28}$ (für 17a) bzw. $\left[\mathrm{Mer}-\mathrm{PPh}_{2}\right]_{2}\left[\mathrm{Pd}\left(\mathrm{N}_{3}\right)_{2}\right]_{0.42}$ (für $\left.\mathbf{1 7} \mathbf{b}-\mathbf{e}\right)$ werden in $20 \mathrm{ml} \mathrm{EtOH}$ mit $100 \mathrm{mg}(2,44 \mathrm{mmol}) \mathrm{CH}_{3} \mathrm{CN}(\mathbf{1 7 a})$, $250 \mathrm{mg} \quad(2,42 \mathrm{mmol}) \quad \mathrm{C}_{6} \mathrm{H}_{5} \mathrm{CN} \quad(\mathbf{1 7 b}), \quad 250 \mathrm{mg}$ $(2,5 \mathrm{mmol}) \quad \mathrm{CH}_{3} \mathrm{O}_{2} \mathrm{CCH}_{2} \mathrm{CN} \quad(\mathbf{1 7} \mathrm{c}), \quad 165 \mathrm{mg}$ $(2,5 \mathrm{mmol}) \quad \mathrm{NC}-\mathrm{CH}_{2}-\mathrm{CN}$ (17 d) bzw. $320 \mathrm{mg}$ $(2,5 \mathrm{mmol}) o-\mathrm{C}_{6} \mathrm{H}_{4}(\mathrm{CN})_{2}(\mathbf{1 7}$ e $) 12 \mathrm{~h}$ unter Rückfluß erhitzt. Die Produkte werden abfiltriert, mit EtOH gewaschen und i. Hochvak. getrocknet.
17a $\left[\mathrm{C}_{63.4} \mathrm{H}_{61.3} \mathrm{Cl}_{0.13} \mathrm{P}\right]_{n}\left[\mathrm{Pd}\left(\mathrm{N}_{4} \mathrm{CCH}_{3}\right)_{2}\right]_{0,28 n / 2}$
Ber. C 85,64 H 6,98 N 1,75, Gef. C 83,89 H 7,22 N 1,90.

17b $\left[\mathrm{C}_{63.4} \mathrm{H}_{61.3} \mathrm{Cl}_{0.13} \mathrm{P}\right]_{n}\left[\mathrm{Pd}\left(\mathrm{N}_{4} \mathrm{CC}_{6} \mathrm{H}_{5}\right)_{2}\right]_{0,42 n / 2}$ Ber. C 84,57 H 6,78 N 2,49, Gef. C 84,59 H 6,94 N 2,22.

$17 \mathbf{c}\left[\mathrm{C}_{63.4} \mathrm{H}_{61.3} \mathrm{Cl}_{0.13} \mathrm{P}\right]_{n}\left[\mathrm{Pd}\left(\mathrm{N}_{4} \mathrm{CCH}_{2} \mathrm{COOCH}_{3}\right)_{2}\right]_{0.26 n / 2}$ Ber. C 85,11 H 6,94 N 1,60, Gef. C 83,12 H 7,19 N 1,61.

$17 \mathbf{d}\left[\mathrm{C}_{63.4} \mathrm{H}_{61.3} \mathrm{Cl}_{0.13} \mathrm{P}\right]_{n}\left[\mathrm{Pd}\left(\mathrm{N}_{4} \mathrm{CCH}_{2} \mathrm{CN}\right)_{2}\right]_{0,42 n / 2}$ Ber. C 83,81 H 6,76 N 3,17, Gef. C 84,77 H 6,90 N 3,27. 
17e $\left[\mathrm{C}_{63.4} \mathrm{H}_{61.3} \mathrm{Cl}_{0.13} \mathrm{P}\right]_{n}\left[\mathrm{Pd}\left(\mathrm{N}_{4} \mathrm{CC}_{6} \mathrm{H}_{4} \mathrm{CN}\right)_{2}\right]_{0,42 n / 2}$

Ber. C 84,17 H 6,66 N 3,08,

Gef. C 83,88 H 6,87 N 2,88.

\section{Polymeres Diphosphan-bis(triazolato)- palladium(II) (18)}

$400 \mathrm{mg}\left[\mathrm{Mer}-\mathrm{PPh}_{2}\right]_{2}\left[\mathrm{Pd}\left(\mathrm{N}_{3}\right)_{2}\right]_{0,42}$ werden in $10 \mathrm{ml}$ $\mathrm{CH}_{3} \mathrm{OH}$ mit $350 \mathrm{mg}(2,5 \mathrm{mmol})$

$\mathrm{CH}_{3} \mathrm{O}_{2} \mathrm{C}-\mathrm{C} \equiv \mathrm{C}-\mathrm{CO}_{2} \mathrm{CH}_{3} 2 \mathrm{~d}$ gerührt. Das hellgelbe Polymere wird abfiltriert, mit $\mathrm{CH}_{3} \mathrm{OH}$ gewaschen und i. Hochvak. getrocknet.

$$
\begin{aligned}
& {\left[\mathrm{C}_{63.4} \mathrm{H}_{61.3} \mathrm{Cl}_{0.13} \mathrm{P}\right]_{n}\left[\mathrm{Pd}\left(\mathrm{N}_{3} \mathrm{C}_{2}\left(\mathrm{COOCH}_{3}\right)_{2}\right)_{2}\right]_{0,42 n / 2}} \\
& \text { Ber. C 84,98 H 6,90 N 1,89, } \\
& \text { Gef. C 82,57 H 6,92 N 1,86. }
\end{aligned}
$$

\section{5-Phenyltetrazol}

Die Suspension von $1 \mathrm{~g} \mathbf{1 7} \mathbf{b}$ in $10 \mathrm{ml} \mathrm{EtOH}$ wird bei R.T. mit $5 \mathrm{ml} \mathrm{HCl-gesättigtem} \mathrm{EtOH}$ versetzt. Augenblicklich tritt die hellgelbe Farbe des Chlorokomplexes auf, der abfiltriert wird. Das Filtrat wurde bis zur Trockene eingeengt, wobei das Tetrazol als farblose Verbindung anfällt. Der Chlorokomplex wird mit Natriumazid zum Azidokomplex umgesetzt, an welchen erneut Benzonitril addiert wird. Das 5-Phenyltetrazol wird wiederum mit ethanolischer $\mathrm{HCl}$ abgespalten usw. Der Zyklus wird noch zweimal wiederholt. Die 5-Phenyltetrazol-Chargen (je $20 \mathrm{mg}$ ) werden vereinigt und aus Ethanol umkristallisiert. Ausbeute: $60 \mathrm{mg}$; Schmp. $209^{\circ} \mathrm{C}$ (Lit.: $\left.212-213^{\circ} \mathrm{C}\right)$.

\section{$\mathrm{C}_{7} \mathrm{H}_{6} \mathrm{~N}_{4}(146,15)$}

$$
\text { Ber. C 57,53 H 4,14 N 38,33, }
$$

Gef. C 56,74 H 4,25 N 37,60.

Die Abspaltung mit Acetylchlorid führt zum extrem hydrolyseempfindlichen 2 Acetyl-5-phenyltetrazol. Ausbeute: $20 \mathrm{mg}$; Schmp. $93{ }^{\circ} \mathrm{C}$ (Lit.: $\left.95-97^{\circ} \mathrm{C}\right)$.

\section{5-(2-Cyanophenyl)tetrazol (8e) aus $\mathbf{1 7} \mathbf{e}$}

Die Abspaltung erfolgt analog zur Abspaltung des 5-Phenyltetrazols (ohne Durchführung weiterer Zyklen). Ausbeute: $10 \mathrm{mg}$.

$$
\begin{array}{rrrrr}
\mathrm{C}_{8} \mathrm{H}_{5} \mathrm{~N}_{5}(171,16) & & \\
\text { Ber. } & \text { C } 56,14 & \text { H } 2,94 & \text { N 40,98, } \\
\text { Gef. } & \text { C } 56,38 & \text { H 3,24 } & \text { N 39,44. }
\end{array}
$$

Röntgenstrukturanalyse von $\mathbf{8 a}$ : Einkristall $0,45 \times 0,3 \times 0,2 \mathrm{~mm} ; \lambda=71,069 \mathrm{pm} ; a=757,8(3)$, $b=904,9(3), c=975,8(4) \mathrm{pm}, \beta=102,00(4)^{\circ}, \mathrm{V}=$ $0,6546(5) \mathrm{nm}^{3}, \mathrm{~d}_{\mathrm{r}}=1,901, \mathrm{~d}_{\exp }=1,89(2) \mathrm{g} / \mathrm{cm}^{3}$ (Flotation in $\mathrm{CCl}_{4} / \mathrm{CHBr}_{3}$ ); Raumgruppe $\mathrm{P}_{2} / c, Z=4$, Datensammlung: $4<2 \theta<43 ; \pm h, \pm k, \pm l ; \omega$-Scan, Breite $1^{\circ}$, Reflex: Untergrund 1:1; Meßgeschwindigkeit $4-29,3^{\circ} / \mathrm{min} ; 2$ Kontrollmessungen alle 48 Intensitätsmessungen; 2083 Reflexe gemessen, 694 unabhängige, beobachtete Reflexe (I $>2 \sigma(\mathrm{I})), 86$ Parameter, $R=0,0385, R_{\mathrm{w}}=0,0437, \mathrm{w}=1 /\left({ }^{2}\left(\mathrm{~F}_{\mathrm{o}}\right)+\right.$ $\left.0,0002 \cdot \mathrm{F}_{\mathrm{o}}^{2}\right)$.

Der Kristall zersetzte sich während der Datensammlung, diese Zersetzung wurde anhand der Standardreflexe korrigiert. Alle Atome bis auf das $\mathrm{H}$ Atom wurden anisotrop verfeinert. Für das Wasserstoffatom wurden die Ortskoordinaten und ein isotroper thermischer Parameter verfeinert. Tab. II enthält die Atomkoordinaten*.

Die Deutsche Forschungsgemeinschaft und der Fonds der Chemischen Industrie haben diese Arbeit großzügig unterstützt, wofür wir herzlich danken. Ebenso danken wir Herrn Dr. E. Leidl und Frau Ch. Fengler für wertvolle Mitarbeit.

\footnotetext{
* Weitere Einzelheiten zur Kristallstrukturuntersuchung können beim Fachinformationszentrum Energie, Physik, Mathematik, D-7514 Eggenstein-Leopoldshafen, unter Angabe der Hinterlegungsnummer CSD 52016, des Autors und des Zeitschriftenzitats angefordert werden.
}

[1] LXIV. Mitteilung: M. Schaal, W. Weigand, U. Nagel und W. Beck, Chem. Ber. 118, 2186 (1985).

[2] W. Beck und W. P. Fehlhammer, Angew. Chem. 79, 146 (1967); Angew. Chem., Int. Ed. Engl. 6, 169 (1967); W. Beck, W. P. Fehlhammer, H. Bock und M. Bauder, Chem. Ber. 102, 3637 (1969); W. Beck, K. Burger und W. P. Fehlhammer, Chem. Ber. 104, 1816 (1971); J. Ch. Weis und W. Beck, Chem. Ber. 105, 3203 (1972); W. P. Fehlhammer, T. Kemmerich und W. Beck, Chem. Ber. 112, 468 (1979); P. H. Kreuzer, J. Ch. Weis, H. Bock, J. Erbe und W. Beck, Chem. Ber. 116; 2691 (1983); J. Erbe und W. Beck, Chem. Ber. 116, 3867 (1983); W. P. Fehlhammer und W. Beck, Z. Naturforsch. 38b, 546 (1983).
[3] R. F. Ziolo, J. A. Thich und Z. Dori, Inorg. Chem. 11, 626 (1972); Z. Dori und R. F. Ziolo, Chem. Rev. 73, 247 (1973); F. Sato, M. Etoh und M. Sato, J. Organomet. Chem. 37, C 51 (1972); 70, 101 (1974); A. Rosan und M. Rosenblum, J. Organomet. Chem. 80, 103 (1974); L. Busetto, A. Palazzi und R. Ros, Inorg. Chim. Acta 13, 233 (1975); W. Rigby, P. M. Bailey, J. A. McCleverty und P. M. Maitlis, J. Chem. Soc. Dalton Trans. 1979, 371; G. La Monica, G. Ardizzoia, S. Cenini und F. Porta, J. Organomet. Chem. 273, 263 (1984).

[4] a) P. Kreutzer, Ch. Weis, H. Boehme, T. Kemmerich, W. Beck, C. Spencer und R. Mason, Z. Naturforsch. 27b, 745 (1972);

b) T. Kemmerich, J. H. Nelson, N. E. Takach, 
H. Boehme, B. Jablonski und W. Beck, Inorg. Chem. 21, 1226 (1982).

[5] R. Huisgen, Angew. Chem. 75, 604, 742 (1963); Angew. Chem., Int. Ed. Engl. 2, 565, 633 (1963); R. Huisgen, in A. Padwa (ed.): 1,3-Dipolar Cycloaddition Chemistry, Wiley-Interscience, New York 1984, Vol. I.

[6] N. E. Takach, E. M. Holt, N. W. Alcock, R. A. Henry und J. H. Nelson, J. Am. Chem. Soc. 102, 2968 (1980).

[7] N. E. Takach und J. H. Nelson, Inorg. Chem. 20, 1258 (1981).

[8] W. Beck, R. Höfer, J. Erbe, H. Menzel, U. Nagel und G. Platzen, Z. Naturforsch. 29b, 567 (1974).

[9] C. S. Marvel und N. Tarköy, J. Am. Chem. Soc. 79, 6000 (1957); 80, 832 (1958); M. N. Patel und S. H. Patil, Synth. React. Inorg. Met.-Org. Chem. 13, 133 (1983).

[10] W. Sawodny, M. Riederer und E. Urban, Inorg. Chim. Acta 29, 63 (1978).

[11] W. Beck, T. Kemmerich und H. Böhme, Z. Naturforsch. 34b, 200 (1979); V. R. Vijayaraghavan, N. Thillaichidambaram, A. Raghavan und M. Santappa, J. Indian Chem. Soc. 55, 532 (1978).

[12] M. N. Ponnuswamy und J. Trotter, Acta Crystallogr. C 39, 726 (1983); J. H. Nelson, N. E. Takach, N. Bresciani-Pahor, L. Randaccio und E. Zangrando, Acta Crystallogr. C 40, 742 (1984).

[13] W. R. Ellis und W. L. Purcell, Inorg. Chem. 21, 834 (1982); W. L. Purcell, Inorg. Chem. 22, 1205 (1983); R. J. Balahura, W. L. Purcell, M. E. Victoriano, M. L. Lieberman, V. M. Loyola, W. Fleming und J. W. Fronabarger, Inorg. Chem. 22, 3602 (1983); J. H. Hall, R. Lopez de la Vega und W. L. Purcell, Inorg. Chim. Acta 102, 157 (1985).

[14] Kurzmitteilung: W. Beck und J. Geisenberger, Chem. \& Eng. News, March 5, 1984, p. 39.

[15] R. K. Howe und M. L. Rueppel, Chem. \& Eng. News, Jan. 17, 1983, p. 4. Ebenso explodiert eine Mischung von $\mathrm{CCl}_{3} \mathrm{CN}$ und $\mathrm{Me}_{3} \mathrm{SiN}_{3}$ beim Erhitzen, während offenbar 1-Trimethylsilyl-5-trichloromethyltetrazol nicht explosiv ist (L. A. Lazukina und V. P. Kukhar, J. Org. Chem. (USSR) 15, 2009 (1979)). Von L. A. Burke (Chem. \& Eng. News, April 25, 1983, p. 2) wird dagegen als Ursache für die beobachteten Explosionen die primäre Bildung des aufgrund theoretischer Berech- nungen instabilen Azidoazomethins, $\mathrm{HN}=\mathrm{C}\left(\mathrm{CCl}_{3}\right) \mathrm{N}_{3}$ (L. A. Burke, J. Elguero, G. Leroy und M. Sana, J. Am. Chem. Soc. 98, 1685 (1976); L. A. Burke, G. Leroy, M. T. Nguyen und M. Sana, J. Am. Chem. Soc. 100, 3668 (1978)) angenommen. Wir danken Herrn Professor L. A. Burke für persönliche Mitteilungen.

[16] R. Huisgen, J. Sauer, H. J. Sturm und J. H. Markgraf, Chem. Ber. 93, 2106 (1960).

[17] Vgl. Lit. [2].

[18] H. Sugimoto, N. Ueda und M. Mori, Bull. Chem. Soc. Jpn. 54, 3425 (1981).

[19] M. Perree-Fauvet, A. Gaudemer, P. Boucly und J. Devynck, J. Organomet. Chem. 120, 439 (1976).

[20] Vgl. z.B. W. Beck und K. Schloter, Z. Naturforsch. 33b, 1214 (1978). In Porphinatoeisen(III)-Komplexen konnte jedoch eine Bindung der schwach koordinierenden Anionen $\mathrm{ClO}_{4}{ }^{-}, \mathrm{BF}_{4}{ }^{-}, \mathrm{PF}_{6}{ }^{-}, \mathrm{SbF}_{6}{ }^{-}$und $\mathrm{CF}_{3} \mathrm{SO}_{3}{ }^{-}$an das Eisenatom eindeutig nachgewiesen werden. T. Mashiko, M. E. Kastner, K. Spartalian, W. R. Scheidt und C. A. Reed, J. Am. Chem. Soc. 100, 6354 (1978); C. A. Reed, T. Mashiko, S. P. Bentley, M. E. Kastner, W. R. Scheidt, K. Spartalian und G. Lang, J. Am. Chem. Soc. 101, 2948 (1979); K. Shelly, T. Bartczak, W. R. Scheidt und C. A. Reed, Inorg. Chem. 24, 4325 (1985); K. Shelly, C. A. Reed, Y. J. Lee und W. R. Scheidt, J. Am. Chem. Soc. 108, 3117 (1986) und dort zit. Lit.

[21] D. A. Summerville, I. A. Cohen, K. Hatano und W. R. Scheidt, Inorg. Chem. 17, 2906 (1978).

[22] Zusammenfassende Darstellung: D. C. Bailey und S. H. Langer, Chem. Rev. 81, 109 (1981).

[23] G. B. Ansell, J. Chem. Soc., Perkin II 1973, 2036.

[24] K. Britts und I. L. Karle, Acta Crystallogr. 22, 308 (1967).

[25] D. S. S. Gowda, R. Rudman und K. Ravindra Acharya, Acta Crystallogr. B 38, 2487 (1982).

[26] G. J. Palenik, Acta Crystallogr. 16, 596 (1963); M. A. Pierce-Butler, Acta Crystallogr. B 38, 2681 (1982).

[27] J. Geisenberger, Dissertation Universität München 1985.

[28] K. Issleib und A. Tzschach, Chem. Ber. 92, 1118 (1954).

[29] W. O. Haag und D. D. Whitehurst, Belg. Patent Nr. 721, 686 (1969). 